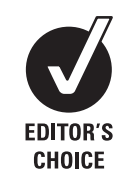

${ }^{1} \mathrm{CHU}$ Clermont-Ferrand, Department of Neurology, Gabriel Montpied Hospital, Clermont-Ferrand, France; ${ }^{2}$ Univ Clermont 1, UFR Medecine, Clermont-Ferrand, France;

${ }^{3}$ INRA, Centre Clermont-Ferrand

Theix, Unite de Nutrition

Humaine, CRNH Auvergne,

Clermont-Ferrand, France; ${ }^{4}$ Univ

Clermont 1, UFR Medecine,

UMR1019, Unite de Nutrition

Humaine, CRNH Auvergne,

CHRU, Clermont-Ferrand,

France; ${ }^{5} \mathrm{CHU}$ Clermont-Ferrand,

Department of Neurosurgery,

Gabriel Montpied Hospital,

Clermont-Ferrand, France

Correspondence to:

S Bannier, Hopital Gabriel

Montpied, Clermont-Ferrand,

France; stephaniebannier@ gmail.com

Received 18 July 2008 Revised 15 October 2008 Accepted 31 October 2008

Published Online First

5 December 2008

\title{
Overweight after deep brain stimulation of the subthalamic nucleus in Parkinson disease: long term follow-up
}

\author{
S Bannier, ${ }^{1,2}$ C Montaurier, ${ }^{3}$ P P Derost, ${ }^{1,2}$ M Ulla, ${ }^{1,2}$ J-J Lemaire, ${ }^{5}$ Y Boirie, ${ }^{3,4}$ B Morio, ${ }^{3}$ \\ F Durif ${ }^{1,2}$
}

\begin{abstract}
Objective: To assess the occurrence of weight gain in patients with Parkinson's disease, with an average 16 months of follow-up after subthalamic nucleus deep brain stimulation.
\end{abstract}

Methods: We used dual $x$ ray absorptiometry to evaluate changes in body weight and body composition in 22 patients with Parkinson's disease (15 men and seven women) before surgery, 3 months after surgery and on average 16 months after surgery.

Results: No patient was underweight before surgery and $50 \%$ were overweight. By contrast, $68 \%$ were overweight or obese 3 months after surgery and $82 \%$ after 16 months $(p<0.001)$. For men, the mean increase in body mass index (BMI) was $1.14(0.23) \mathrm{kg} / \mathrm{m}^{2} 3$ months after surgery and $2.02(0.36) \mathrm{kg} / \mathrm{m}^{2} 16$ months after surgery. For women, the mean increases in $\mathrm{BMI}$ at the same evaluation times were $1.04(0.30) \mathrm{kg} / \mathrm{m}^{2}$ and 2.11 $(0.49) \mathrm{kg} / \mathrm{m}^{2}$. This weight gain was mainly secondary to an increase in fat mass in both men and women. Three months after surgery, acute subthalamic deep brain stimulation induced an improvement in parkinsonian symptoms (evaluated by the Unified Parkinson Disease Rating Scale (UPDRS) part III) by 60.7 (2.9)\% in the "off" dopa condition and a dramatic improvement of motor complications (dyskinesia duration: 82.8 (12.8)\%, $\mathrm{p}<0.0001$; off period duration: 92.7 (18.8)\%, $\mathrm{p}<0.0001$ ). Conclusion: Although subthalamic nucleus deep brain stimulation significantly improved parkinsonian symptoms and motor complications, many patients became overweight or obese. This finding highlights the necessity to understand the underlying mechanisms and to provide a diet management with a physical training schedule appropriate for patients with Parkinson's disease.

For several years subthalamic nucleus deep brain stimulation (STN DBS) has proved to be an effective safe procedure ${ }^{1-5}$ for patients with advanced Parkinson's disease (PD). It permits a dramatic improvement in parkinsonian symptoms (eg, akinesia, rigidity, tremor and motor complications such as levodopa induced dyskinesia (LID) and "off" period symptoms). It also allows a significant reduction in levodopa therapy.

In the past 10 years, numerous teams have reported marked gain in weight after STN DBS. ${ }^{6-10}$ In a previous work, ${ }^{10}$ we showed a mean body weight gain of $3.4(0.6) \mathrm{kg}(-1.5$ to $+6.8 \mathrm{~kg})$ 3 months after STN DBS. We also showed in this study that patients' daily energy expenditure (evaluated in a calorimetric chamber) was significantly reduced after STN DBS (-8.98
$(1.72) \%)$ with no change in food intake. This rapid massive body weight gain 3 months after surgery was also demonstrated by other teams ${ }^{6} 79$ but to date no prospective study has been performed to examine long term changes in body weight of patients with $\mathrm{PD}$ and to assess whether the occurrence of overweight or obesity could be a major concern.

Accordingly, we undertook a study to evaluate body weight changes in patients with $\mathrm{PD}$ in the long term after surgery. Hence we measured body weight and composition using dual $x$ ray absorptiometry before surgery, 3 months after surgery and on average 16 months after STN DBS in 22 men and women with PD.

\section{METHODS}

\section{Subjects}

A total of 22 patients with PD, aged 60.5 (1.4) years, were included, comprising 15 men and seven women. History of disease averaged 9.8 (0.6) years. All patients were suffering from idiopathic PD according to the criteria of the Parkinson's Disease Society Brain Bank. ${ }^{11}$ They all described severe motor fluctuations and LID that were not improved by optimal antiparkinsonian medication. Selection criteria for surgery were: an excellent response to levodopa, tested during an acute levodopa challenge; no postural instability during the best "on" period; no dementia (Mini Mental Scale $>24$, Mattis scale $>130 / 144$ ); and normal magnetic resonance imaging. Exclusion criteria for surgery were: psychosis, neoplasia and severe depression. For the specific tool of this study, exclusion criteria were diabetes and thyroid disease. All women were post-menopausal. The study protocol was approved by the regional medical school ethics committee. It was performed according to the principles set out in the declaration of Helsinki and complied with French legislation (the Huriet law).

\section{General study design}

After medical check-ups at inclusion, patients were systematically studied 1 month before surgery $(M-1)$ and 3 months after surgery $(M+3)$. Of the 22 enrolled patients, 15 underwent evaluation 1 year after surgery (eight men and seven women), and seven 2 years after surgery (all men). On average, the second postoperative evaluation was performed 16 months after STN DBS $(M+16)$. 
Table 1 Physical characteristics of the patients with Parkinson's disease before surgery

\begin{tabular}{lcc}
\hline & Men (n= 15) & Women (n=7) \\
\hline Age (years) & $60.1(1.8)$ & $61.4(2.4)$ \\
Height (cm) & $174.8(1.6)$ & $160.0(1.9)$ \\
Body weight (kg) & $75.6(1.8)$ & $62.4(5.4)$ \\
BMI (kg/m²) & $25.3(0.8)$ & $23.7(1.8)$ \\
Fat free mass (kg) & $59.2(1.4)$ & $42.0(1.8)$ \\
Fat mass (kg) & $16.5(1.1)$ & $20.4(3.6)$ \\
Fat mass (\%) & $21.6(1.2)$ & $31.2(3.1)$ \\
History of disease (years) & $9.6(0.6)$ & $10.1(0.8)$ \\
LEDD (mg/day) & $1202.5(91.1)$ & $881.2(56.4)$ \\
UPDRS III improvement* $(\%)$ & $78.8(2.2)$ & $81.7(1.7)$ \\
Mattis score & $138.0(3.9)$ & $137.8(4.8)$ \\
\hline
\end{tabular}

*During an acute levodopa challenge.

BMI, body mass index; LEDD, levodopa equivalent daily dose; UPDRS, Unified Parkinson Disease Rating Scale.

\section{Assessment of parkinsonian symptoms}

One month before surgery, response to levodopa was evaluated in the morning using the Unified Parkinson Disease Rating Scale (UPDRS) part III' in the "off" state after a $12 \mathrm{~h}$ withdrawal of antiparkinsonian medication and after taking 1.5 times the usual morning levodopa dose using dispersible levodopa (Modopar Dispersible; Roche, Basel, Switzerland). The other tests included UPDRS parts I and II ("on" and "off" state), part IV and classification by Hoehn and Yahr stage. Three and, on average, 16 months after surgery, motor symptoms were assessed according to the medication (Med "on"/Med "off") and the stimulation (Stim "on"/Stim "off") conditions. The same further tests as those carried out preoperatively were also performed. The doses of antiparkinsonian medications were expressed as levodopa equivalent daily dose (LEDD).

\section{Body weight}

At $M-1$ and $M+16$, body mass was measured to the nearest $0.1 \mathrm{~kg}$ on SECA 709 scales (SECA, Les Mureaux, France). Height was measured to the nearest $0.2 \mathrm{~cm}$. Body mass index (BMI) was calculated from height and weight $\left(\mathrm{kg} / \mathrm{m}^{2}\right)$.

A total body scan was performed using DEXA (Hologic ODR 4501; Hologic Inc, Walthan, USA) to determine total and regional body composition (arms, legs and trunk). Fat free mass (FFM) was calculated as the sum of lean mass, soft tissue and bone mineral content. FFM after surgery was corrected for the presence of the stimulator box (FFM was cut for $40 \mathrm{~g}$ ).

\section{Surgery}

The surgical procedure was based on the direct location of STN using stereotactic MRI and electrophysiological mapping (recording and acute stimulation of the STN area) and has been described elsewhere. ${ }^{13}$ One contact of the DBS electrode (DBS Medtronic 3389; Medtronic, Minneapolis, USA) was placed in each hemisphere where the best efficacy of the acute stimulation was found. A few days later the electrodes were connected to a pulse generator (Kinetra; Medtronic). Stimulation settings and antiparkinsonian therapy were adapted postoperatively according to the efficacy of chronic stimulation.

\section{Statistical analysis}

Results are reported as means (SEM). The variations in weight $(\mathrm{kg})$ and $\mathrm{BMI}\left(\mathrm{kg} / \mathrm{m}^{2}\right)$ were calculated as percentages, and patient height was considered to be constant over time. Thus the same degree of significance was found between variations in weight and

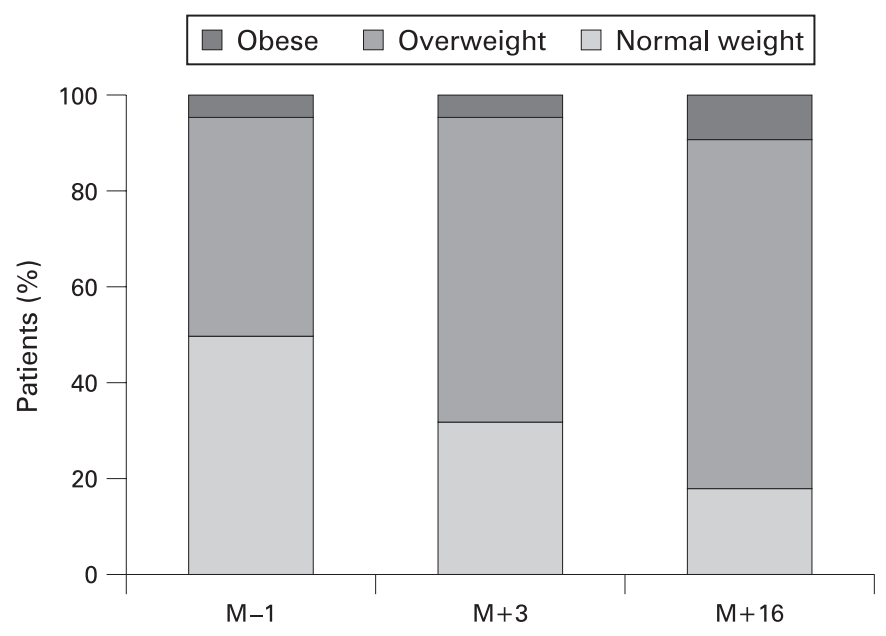

Figure 1 Distribution of weight in our population 1 month before surgery $(M-1), 3$ months after surgery $(M+3)$ and 16 months after surgery $(\mathrm{M}+16)$. Normal weight, $18 \leqslant$ body mass index (BMI)

$<25 \mathrm{~kg} / \mathrm{m}^{2}$; overweight, $25 \leqslant \mathrm{BMl}<30 \mathrm{~kg} / \mathrm{m}^{2}$; obese BMl $\geqslant 30 \mathrm{~kg} / \mathrm{m}^{2}$.

The number of patients who were overweight $(B M I \geqslant 25)$ increased statistically over the three successive observation times $(p<0.001)$.

variations in BMI. For the multiple comparison of data between $M-1, M+3$ and $M+16$, we used Friedman variance analysis. When the difference observed was significant, we studied differences between groups with a Wilcoxon test. In this case, a Bonferroni correction was necessary with p significant when less than 0.017 . Correlations were Pearson product moment correlations with results considered statistically significant at the $5 \%$ level. A $\chi^{2}$ test was performed to analyse the distribution of the patients in two different groups (normal weight or overweight) over time. Statistics were analysed using Statview 5.0 software (SAS Institute Inc., Cary, North Carolina, USA).

\section{RESULTS}

The clinical characteristics of the patients included are summarised in table 1.

\section{Changes in body weight}

Using BMI, we classified patients (both men and women) into underweight $\left(\mathrm{BMI}<18 \mathrm{~kg} / \mathrm{m}^{2}\right)$, normal weight $(18 \leqslant \mathrm{BMI}$ $\left.<25 \mathrm{~kg} / \mathrm{m}^{2}\right)$, overweight $\left(25 \leqslant\right.$ BMI $\left.<30 \mathrm{~kg} / \mathrm{m}^{2}\right)$ and obese $\left(\mathrm{BMI}>30 \mathrm{~kg} / \mathrm{m}^{2}\right)$.

Before surgery, none of the patients was underweight and $50 \%$ were overweight $(n=11 / 22)$. Three months after DBS, $68 \%$ of patients $(n=14 / 22)$ were overweight or obese. This rate increased to $82 \%(n=18 / 22)$ at the last follow-up 16 months after surgery. Two patients did not gain weight: a 57 -year-old man with a preoperative BMI of $29.4 \mathrm{~kg} / \mathrm{m}^{2}$ and a 69 -year-old man with a preoperative BMI of $28.4 \mathrm{~kg} / \mathrm{m}^{2}$. Ten patients had a total weight gain at 16 months higher than $10 \%$ over their preoperative weight. A $\chi^{2}$ test confirmed that the number of patients who were overweight increased significantly with time $(p<0.001)$ (fig 1).

For men, there was significant weight gain $(p<0.001)$. Body weight gain averaged $3.5(0.7) \mathrm{kg}(\mathrm{p}<0.001)$ at $\mathrm{M}+3$ and 6.2 (1.1) $\mathrm{kg}(\mathrm{p}<0.0001)$ at $\mathrm{M}+16$ compared with preoperative data. Mean BMI was $24.9(0.6) \mathrm{kg} / \mathrm{m}^{2}$ at $\mathrm{M}-1,26.0(0.5) \mathrm{kg} / \mathrm{m}^{2}$ at $\mathrm{M}+3$ and $26.9(0.6) \mathrm{kg} / \mathrm{m}^{2}$ at $\mathrm{M}+16$.

For women, a Friedman test showed a significant weight gain $(p=0.01)$ but because of the small size of the sample, 

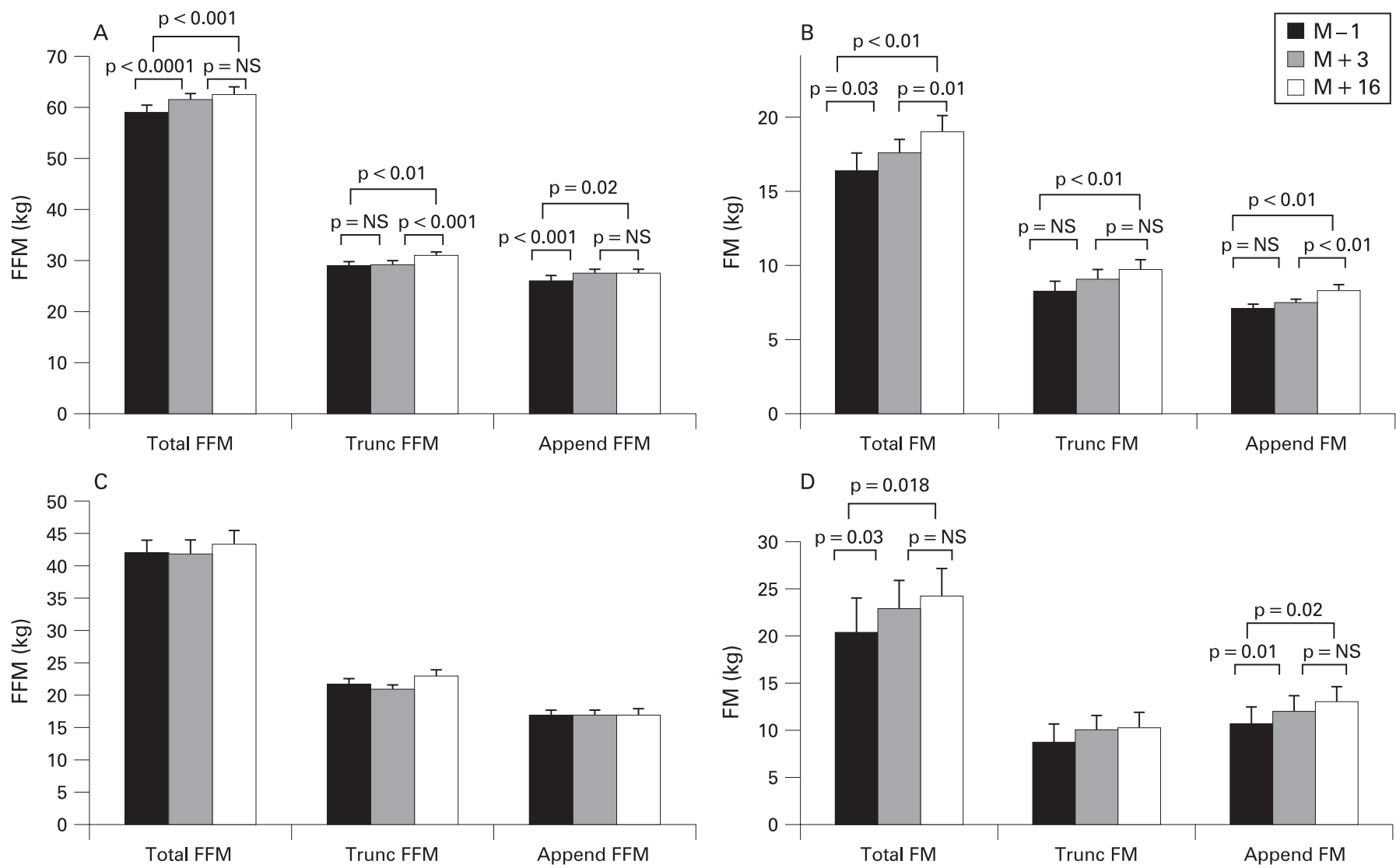

Figure 2 (A) Distribution of fat free mass (FFM) for men. There was a significant increase in total FFM $(p<0.001)$, truncular (trunc) FFM ( $<<0.0001)$ and appendicular (append) FFM $(\mathrm{p}<0.01)$. A significant increase in appendicular FFM (append FFM) was initially observed between $M-1$ (1 month before surgery) and $M+3$ ( 3 months after surgery) whereas we only observed an increase in truncular FFM (trunk FFM) between $M+3$ and $M+16$ (16 months after surgery). (B) Distribution of fat mass (FM) for men. There was a significant increase in total FM ( $<<0.01)$, truncular FM ( $p<0.01)$ and appendicular FM $(\mathrm{p}<0.001)$. Post hoc analysis showed significant increases in both appendicular and truncular fat mass between $\mathrm{M}-1$ and $\mathrm{M}+16$. (C) Distribution of FFM for women. There was no variation in FFM for appendicular FFM or for truncular FFM. (D) Distribution of FM for women. Despite the sample being small, a significant increase in FM was observed $(p=0.01)$ in appendicular FM $(p<0.01)$ but not in truncular FM. Post hoc analysis did not find any significant difference.

one-to-one comparison was not significant after Bonferroni correction: mean weight gain averaged $2.6(0.8) \mathrm{kg}(\mathrm{p}=0.018)$ at 3 months and $5.5(1.2) \mathrm{kg}(\mathrm{p}=0.028)$ at 16 months. Mean BMI was $24.2(1.8) \mathrm{kg} / \mathrm{m}^{2}$ at $\mathrm{M}-1,25.3(1.5) \mathrm{kg} / \mathrm{m}^{2}$ at $\mathrm{M}+3$ and $26.4(1.4) \mathrm{kg} / \mathrm{m}^{2}$ at $\mathrm{M}+16$.

\section{Changes in body composition}

For men, FFM significantly increased by $3.9 \quad(0.7) \%$ $(p<0.0001)$ at 3 months and by $5.9(1.3) \%(p<0.001)$ at 16 months (fig 2A). For appendicular FFM (muscular mass), post hoc analysis showed that a significant increase occurred only at $M+3$. Between $M+3$ and $M+16$, we observed an increase in the FFM for men but only in the truncular compartment (visceral FFM). The fat mass increased by 9.2 (3.3) \% at 3 months ( $p=0.03$; NS after Bonferroni correction) and by $19.5(5.2) \%$ at 16 months $(p<0.01)$. This fat mass increase was observed in both the appendicular and truncular compartments (fig 2B).

For women, FFM did not change after DBS (fig 2C). In contrast, fat mass significantly increased ( $p=0.01$ ) (fig 2D). After post hoc analysis, fat mass increased by 20 (8)\% at 3 months ( $p=0.03$, NS after Bonferroni correction) and by 30.2 $(14.1) \%$ at $M+16(p=0.018)$. The increase in fat mass was found for the appendicular compartment $(p<0.01)$ but was not significant for the truncular compartment $(p=0.09)$.
Body weight gain was closely correlated with a low preoperative weight $(r=0.57 ; p<0.0001)$ (fig $3 \mathrm{~A})$. Conversely, a high rate of weight gain during the first 3 months after surgery was not predictive of further high weight gain at the $M+16$ evaluation.

A significant correlation was found between fat mass variation and UPDRS part III variation while stimulation was "on". In fact, UPDRS part III improvement was correlated with a lower trend in fat mass increase. This was true at both $M+3$ and $M+16(r=0.49$; $p<0.05)$. This correlation was only found with fat mass variation (fig $3 \mathrm{~B})$. There was no correlation between total weight variation and UPDRS part III variation and no correlation between motor complications such as change in "off" period duration, severity and duration of LID, LEDD and weight gain.

\section{Surgery outcomes}

\section{Acute effects of DBS-STN}

Three months after surgery, STN DBS when turned "on" (in the "off" levodopa condition) gave 60.7 (2.9)\% improvement in the UPDRS part III. Sixteen months after surgery, DBS still produced 52.4 (3.1)\% improvement (in the "off" levodopa condition). Tremor, assessed from the subscores of the UPDRS part III, was significantly decreased with STN DBS by 90.1 $(21.1) \%(p<0.001) 3$ months after surgery and by $96.8(20.9) \%$ $(p<0.001) 16$ months after surgery. Similarly, akinesia improved by $53.3(7.8) \%(p<0.0001) 3$ months after STN-DBS 

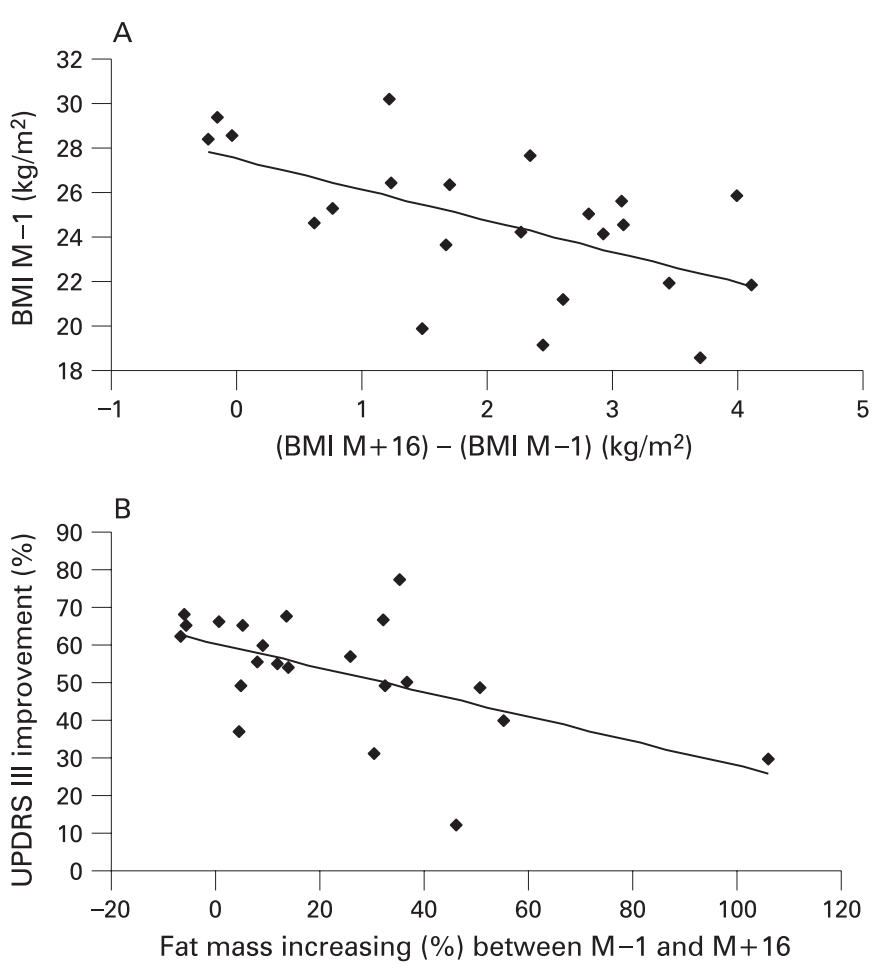

Figure 3 (A) Correlation between preoperative body mass index (preop $\mathrm{BMI})$ and variation in $\mathrm{BMI}(\mathrm{BMI}(\mathrm{M}+16)-\mathrm{BMI}(\mathrm{M}-1))$. There was a significant relation between a low preoperative $\mathrm{BMI}$ and the magnitude of the gain in BMI $(r=0.57 ; p<0.01)$. (B) Correlation between improvement in Unified Parkinson Disease Rating Scale (UPDRS) part III motor score with stimulation turned "on" (in the off dopa condition) and variation in fat mass. Improvement in UPDRS III was correlated $(r=0.56 ; p<0.01)$ in a negative relationship with the reduction in BMI. $M-1,1$ month before surgery; $M+3,3$ months after surgery; $M+16$, 16 months after surgery.

and by $53.1(4.5) \%(p<0.0001) 16$ months after surgery. Finally, rigidity was ameliorated by $64.2(6.9) \%(p<0.0001)$ and 56.3 $(5.0) \%(p<0.0001)$ at $M+3$ and $M+16$ after STN DBS (table 2$)$.

\section{Chronic effect of DBS-STN}

Motor complications, assessed by UPDRS part IV, dramatically improved. Duration of LID decreased by $82.8(12.8) \%(p<0.001)$ and $93.9(16.6) \%(p<0.0001)$ at $M+3$ and $M+16$. Severity of LID improved by $89.0 \quad(18.5) \% \quad(p<0.001)$ and $92.7 \quad(18.8) \%$ $(p<0.0001)$ at $M+3$ and $M+16$. Finally, the duration of the "off" period decreased by $64.7(10.0) \%(p<0.0001)$ and 94.2 (9.6)\% $(p<0.0001)$ at $M+3$ and $M+16$.

The UPDRS part II score significantly decreased after STN DBS in the "off" medication conditions: -40.6 (7.7)\% $(p<0.0001)$ at $M+3$ and $-27.8(8.1) \%(p<0.001)$ at $M+16$. In the "on" medication condition, UPDRS II worsened: the raw scores were $3.5(0.7)$ at $M-1$ and $5.6(0.8)$ at $M+3(p=0.019)$ and 7.9 (0.6) at $M+16(p<0.0001$ between $M-1$ and $M+16)$ (table 2). LEDD decreased by $39(5) \%(p<0.0001)$ and $28(6) \%$ $(p<0.01)$ from baseline to $M+3$ and $M+16$ (table 2). The stimulation settings were $2.7(0.1)$ and $2.8(0.1) \mathrm{V}$ (right and left sides), 148.0 (4.5) $\mathrm{Hz}$ and 69.0 (5.3) $\mu$ s at $\mathrm{M}+3$. At $\mathrm{M}+16$, the stimulation settings were unchanged: $2.7(0.1)$ and $2.8(0.1) \mathrm{V}$ (right and left sides), 148.0 (4.5) $\mathrm{Hz}$ and 68.0 (2.9) $\mu \mathrm{s}$.

\section{DISCUSSION}

In this study, very rapid weight gain was observed during the first 3 months after surgery. Men gained $3.5(0.7) \mathrm{kg}$ and women $2.6(0.8) \mathrm{kg} 3$ months after surgery. During this period, men gained FFM (mainly muscle mass) as well as fat mass whereas women only gained fat mass. Such a fast gain of weight has been validated by other teams. ${ }^{679}{ }^{14}$ However, the weight gain continued after the first 3 months, leading to a serious risk of overweight. During this time, both men and women gained only fat mass and truncular FFM. No change in appendicular FFM (muscle mass) was observed. The major studies on weight gain in PD patients after STN DBS considered that this effect was a homeostatic response to the previous disease related weight loss. ${ }^{6}{ }^{14}$ Thus it was thought that patients with PD normalised their weight relative to their premorbid status. However, we showed here a massive weight gain at 16 months evaluated at $6.2(1.0) \mathrm{kg}$ for men and $5.5(1.2) \mathrm{kg}$ for women. Furthermore, more than $80 \%$ of patients were overweight at this time. These results show that weight gain is not only normalisation of preoperative weight but also an excessive weight gain which can be life threatening and markedly increases cardiovascular risk. ${ }^{15}$

The distribution of these weight changes was quite different between men and women. It is likely that the initial gain in muscle mass for men was secondary to the increase in physical activity after STN DBS. The gain in muscular mass remained over time but did not go on increasing, unlike fat mass and

Table 2 Assessment of the clinical variables of the parkinsonian patients

\begin{tabular}{lcccl}
\hline & M-1 & M+3 & M+16 & p Value* \\
\hline LEDD (mg/day) & $1135.4(91.4)$ & $622.4(55.4)$ & $741.1(68.5)$ & $<0.001$ \\
UPDRS III M "off"/S "off" & $31.5(2.0)$ & $33.8(3.0)$ & $34.4(1.7)$ & NS \\
UPDRS III M "off"/S "on" & $12.9(1.5)$ & $16.3(1.4)$ & NS & \\
UPDRS III M "on"/S "off" & $6.5(0.8)$ & $11.0(1.3)$ & $9.3(0.9)$ & NS \\
UPDRS III M "on" S "on" & $6.8(0.9)$ & $8.9(0.9)$ & NS & \\
Hoehn and Yahr "off" & $2.4(0.1)$ & $1.9(0.1)$ & $2.3(0.1)$ & NS \\
Hoehn and Yahr "on" & $1.7(0.2)$ & $1.5(0.2)$ & $1.6(0.2)$ & NS \\
LID duration & $1.5(0.2)$ & $0.3(0.1)$ & $0.1(0.1)$ & $<0.001$ \\
LID severity & $1.3(0.2)$ & $0.1(0.1)$ & $0.1(0.1)$ & $<0.01$ \\
"Off" period duration & $1.6(0.1)$ & $0.6(0.1)$ & $0.9(0.1)$ & $<0.01$ \\
UPDRS I & $1.3(0.2)$ & $1.1(0.2)$ & $1.6(0.4)$ & NS \\
UPDRS II "off" & $17.0(0.8)$ & $10.11(1.0)$ & $12.3(0.9)$ & $<0.001$ \\
UPDRS II "on" & $3.5(0.7)$ & $5.6(0.8)$ & $7.9(0.6)$ & $<0.001$
\end{tabular}

$\mathrm{p}$ Values were calculated by Friedman analysis for multiple comparisons.

LID, levodopa induced dyskinesia; LEDD, levodopa equivalent daily dose; M, medication; S, stimulation; UPDRS, Unified Parkinson Disease Rating Scale. 
truncular FFM, which continued to increase in both men and women. The fact that patients continued to gain weight in the long term could be linked to a chronic effect of STN DBS on structures that regulate energy expenditure, such as the lateral hypothalamus. We can speculate a regional effect of DBS on the hypothalamus, part of the volume of tissue directly activated by the stimulation. ${ }^{16}$ The alternative hypothesis is that tracts going to or from the hypothalamus could be disrupted after both STN and pallidal DBS and pallidotomy also. ${ }^{17}$ In our study, BMI and the percentage of body fat mass in patients with PD before STN DBS was similar to a control healthy group. ${ }^{10}$ These results are close to those found in other studies on body composition using dual $x$ ray absorptiometry in $\mathrm{PD} .{ }^{18}$ They are consistent with the results of another work ${ }^{19}$ that showed, in addition, that patients with PD only began to lose weight when they developed LID. It seems that even if patients with PD begin to loose weight early in the course of their disease, ${ }^{20}$ a real underweight is not to be feared before several years of disease duration ${ }^{218}$ with clinical complications such as dementia and "axial" signs. Thus it is not uncommon that patients with $\mathrm{PD}$, at the time of surgery, have a normal or excessive $\mathrm{BMI}^{6}{ }^{9}$ (PD patients must have no cognitive impairment or postural instability to be included for STN DBS). In addition, although no patient was underweight before surgery, the lower their weight was before STN DBS, the more they gained weight after surgery.

The benefits of STN DBS for patients with PD in terms of improvement in motor scores and therapy complications were consistent with the literature. This suggests that our population was representative of the general PD population submitted for STN DBS. ${ }^{12}{ }^{21-23}$ The improvement in UPDRS motor score after STN DBS seems to be a protective factor for weight gain. Until now, the major studies about weight gain after STN DBS ${ }^{6}$ failed to find any correlation with UPDRS III improvement. On the other hand, the gain in weight after unilateral pallidotomy ${ }^{17}$ was correlated with UPDRS III improvement in PD. The authors also found a gain in weight after pallidotomy for generalised dystonia; this suggests that UPDRS III improvement in the surgical treatment for $\mathrm{PD}$ is not the only mechanism responsible for weight gain. Another report ${ }^{14}$ found a correlation between weight gain and UPDRS III improvement but the population studied was very heterogeneous in terms of surgical procedure (nine unilateral pallidotomy, nine bilateral pallidal DBS, nine bilateral STN DBS). In contrast, our results suggest that when motor symptoms such as akinesia, rigidity and tremor are substantially alleviated after surgery, patients can recover enough physical activity to increase the energy expenditure related to physical activity. This may be why these patients do not gain so much weight. Interestingly, the two patients who did not gain weight in our study were among those with the higher rate of UPDRS III improvement after DBS ( $62 \%$ and $65 \%$ in the off dopa condition at the $M+16$ evaluation). The impact of the decrease in "off" period symptoms and the improvement in LID on the decrease in energy expenditure remains uncertain. We found no correlation between UPDRS part IV subscores related to LID duration and severity, "off" period duration and weight gain. However, in patients with Huntington's disease, ${ }^{24}$ there is a strong correlation between chorea score (chorea rating scale, range 0-28) and energy expenditure (evaluated in the calorimetric chamber and also in free living conditions). Assessment of motor complications using UPDRS part IV scale in PD was probably not extensive enough to find statistically significant results in the search for correlations with energy expenditure or weight changes. Further studies are being initiated to make a more detailed determination of the impact of STN DBS on changes in free living physical activity level and energy expenditure. Motor complications will be evaluated by means of a diary in which patients themselves note their motor status throughout the day.

In conclusion, all patients undergoing STN surgery should be advised that they are liable to become overweight after chronic DBS. In addition, an initial low weight gain 3 months after surgery is not predictive of a further low weight gain as their was no correlation between the initial weight change 3 months after surgery and that observed 16 months afterwards. Therefore, dieticians and physicians should not limit their nutritional advice to the heaviest patients or to those who gained most weight 3 months after surgery. We advocate offering an overall programme developed with diet management and a physical training schedule for patients undergoing STN DBS.

\section{Competing interests: None.}

Ethics approval: The study was approved by the regional medical school ethics committee.

\section{REFERENCES}

1. Derost PP, Ouchchane L, Morand D, et al. Is DBS-STN appropriate to treat severe Parkinson disease in an elderly population? Neurology 2007:68:1345-55.

2. Lorefalt B, Ganowiak W, Palhagen S, et al. Factors of importance for weight loss in elderly patients with Parkinson's disease. Acta Neurol Scand 2004;110:180-7.

3. Krack P, Batir A, Van Blercom N, et al. Five-year follow-up of bilateral stimulation of the subthalamic nucleus in advanced Parkinson's disease. N Engl J Med 2003;349:1925-34.

4. Volkmann J. Update on surgery for Parkinson's disease. Curr Opin Neurol 2007;20:465-9.

5. Deuschl G, Schade-Brittinger C, Krack $P$, et al. A randomized trial of deep-brain stimulation for Parkinson's disease. N Engl J Med 2006;355:896-908.

6. Barichella $\mathbf{M}$, Marczewska $\mathrm{AM}$, Mariani $\mathbf{C}$, et al. Body weight gain rate in patients with Parkinson's disease and deep brain stimulation. Mov Disord 2003;18:1337-40.

7. Macia F, Perlemoine C, Coman I, et al. Parkinson's disease patients with bilateral subthalamic deep brain stimulation gain weight. Mov Disord 2004;19:206-12.

8. Novakova L, Ruzicka E, Jech R, et al. Increase in body weight is a non-motor side effect of deep brain stimulation of the subthalamic nucleus in Parkinson's disease. Neuro Endocrinol Lett 2007;28:21-5.

9. Tuite PJ, Maxwell RE, lkramuddin S, et al. Weight and body mass index in Parkinson's disease patients after deep brain stimulation surgery. Parkinsonism Relat Disord 2005;11:247-52.

10. Montaurier C, Morio B, Bannier S, et al. Mechanisms of body weight gain in patients with Parkinson's disease after subthalamic stimulation. Brain 2007;130:1808-18.

11. Gibb WR, Lees AJ. The relevance of the Lewy body to the pathogenesis of idiopathic Parkinson's disease. J Neurol Neurosurg Psychiatry 1988;51:745-52.

12. Fraix V, Houeto $\mathrm{JL}$, Lagrange $\mathrm{C}$, et al. Clinical and economic results of bilateral subthalamic nucleus stimulation in Parkinson's disease. J Neurol Neurosurg Psychiatry 2006;71:443-9

13. Lemaire JJ, Coste $J$, Ouchchane L, et al. Brain mapping in stereotactic surgery: a brief overview from the probabilistic targeting to the patient-based anatomic mapping. Neuroimage 2007;37(Suppl 1):S109-15.

14. Gironell A, Pascual-Sedano B, Otermin $P$, et al. Weight gain after functional surgery for Parkinsons disease. Neurologia 2002;17:310-16.

15. Melanson KJ, McInnis KJ, Rippe JM, et al. Obesity and cardiovascular disease risk: research update. Cardiol Rev 2001;9:202-7.

16. Butson CR, Cooper SE, Henderson JM, et al. Patient-specific analysis of the volume of tissue activated during deep brain stimulation. Neuroimage 2007;34:661-70.

17. Ondo WG, Ben-Aire L, Jankovic J, et al. Weight gain following unilateral pallidotomy in Parkinson's disease. Acta Neurol Scand 2000;101:79-84.

18. Beyer PL, Palarino MY, Michalek D, et al. Weight change and body composition in patients with Parkinson's disease. J Am Diet Assoc 1995;95:979-83.

19. Revilla M, Jimenez-Jimenez FJ, Villa LF, et al. Body composition in Parkinson's disease: a study with dual-energy X-ray absorptiometry. Parkinsonism Relat Disord 1998:137-42.

20. Chen H, Zhang SM, Hernan MA, et al. Weight loss in Parkinson's disease. Ann Neurol 2003;53:676-9.

21. Kleiner-Fisman G, Fisman DN, Sime E, et al. Long-term follow up of bilateral deep brain stimulation of the subthalamic nucleus in patients with advanced Parkinson disease. J Neurosurg 2003;99:489-95.

22. Ostergaard K, Aa Sunde N. Evolution of Parkinson's disease during 4 years of bilateral deep brain stimulation of the subthalamic nucleus. Mov Disord 2006;21:624-31.

23. Rodriguez-Oroz MC, Obeso JA, Lang $A E$, et al. Bilateral deep brain stimulation in Parkinson's disease: a multicentre study with 4 years follow-up. Brain 2005:128:2240-9.

24. Pratley RE, Salbe AD, Ravussin E, et al. Higher sedentary energy expenditure in patients with Huntington's disease. Ann Neurol 2000;47:64-70. 